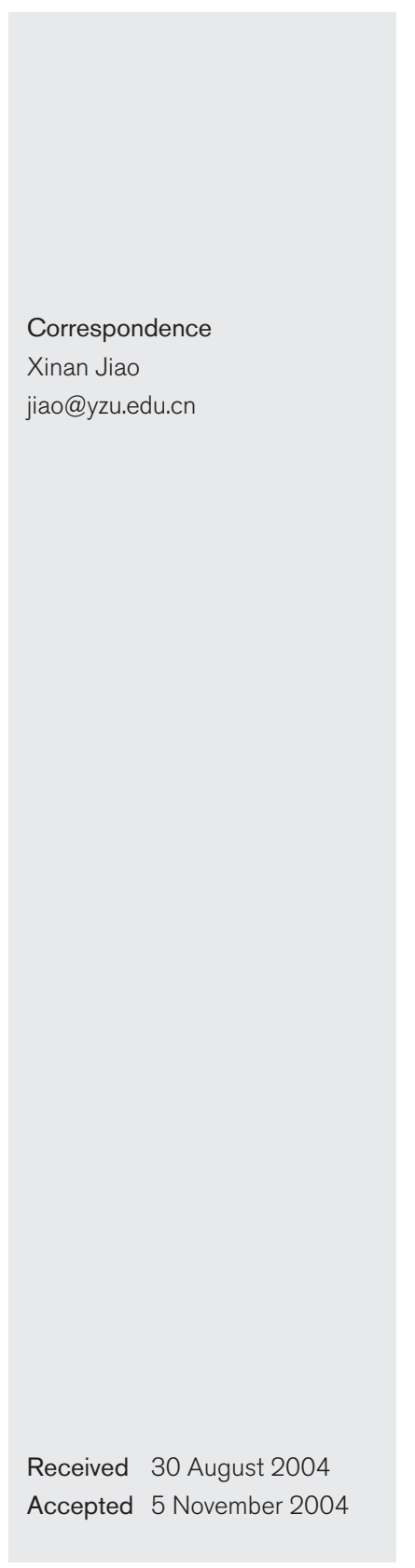

\title{
Listeria monocytogenes in the Chinese food system: strain characterization through partial act $A$ sequencing and tissue-culture pathogenicity assays
}

\author{
Xiaohui Zhou, ${ }^{1}+$ Xinan Jiao ${ }^{1}$ and Martin Wiedmann ${ }^{2}$ \\ ${ }^{1}$ College of Bioscience and Biotechnology, Yangzhou University, 225009, PR China \\ ${ }^{2}$ Department of Food Science, Cornell University, Ithaca, NY 14853, USA
}

\begin{abstract}
Human listeriosis is generally caused by consumption of ready-to-eat (RTE) foods that are stored for extended periods of time at refrigeration temperatures and that permit the growth of the causative agent, Listeria monocytogenes. Food-consumption patterns in China are undergoing rapid changes and more regular consumption of refrigerated-storage RTE foods may increase the risk of human listeriosis. In total, 40 L. monocytogenes isolates were obtained from food $(n=32)$ and sewage $(n=6)$ samples and from two human listeriosis cases that occurred in China. All isolates were characterized into molecular subtypes by DNA sequencing of the $597 \mathrm{bp} \mathrm{3'-terminal} \mathrm{region} \mathrm{of} \mathrm{the}$ virulence gene actA. Sequence data were used to classify the 40 Chinese $L$. monocytogenes isolates into sequence types and phylogenetic lineages, and to compare the sequence types of the Chinese isolates with those of isolates from the USA. Phylogenetic analyses showed that the Chinese isolates could be separated into two genetic lineages, with 14 and 26 isolates belonging to lineages I and II, respectively. Lineage II could be subdivided further into two clusters, IIA and IIB. Lineages I and II were identical to the two lineages described previously among US L. monocytogenes isolates. In total, 14 act $A$ sequence types could be differentiated among the 40 Chinese $L$. monocytogenes isolates; two specific actA sequence types were found among both Chinese and US isolates. Isolates belonging to lineage II showed a significantly lower ability to invade and multiply within human intestinal epithelial Caco- 2 cells than lineage I isolates. It was concluded that DNA sequencing of the $3^{\prime}$-terminal region of act $A$ appears to be an effective method for rapid subtype and lineage classification of $L$. monocytogenes. As strains belonging to lineages I and II have previously been found among isolates from Europe and North America, these results show that $L$. monocytogenes clonal groups found in China are very similar to those found in the USA. Many L. monocytogenes strains may thus represent globally distributed clonal types. Together with the first description of two human listeriosis cases in China, these data indicate that changes in fooddistribution and -consumption patterns in China and other countries will probably lead to the emergence of human listeriosis as a food-safety issue, as virulent strains of this pathogen appear to be present in the Chinese food supply.
\end{abstract}

\section{INTRODUCTION}

Listeria monocytogenes is a facultative, intracellular, Grampositive bacterium that can cause severe food-borne illness in immunocompromised patients, pregnant women and newborns. Symptoms of listeriosis include encephalitis, meningitis and stillbirths (Altimira et al., 1999; Vázquez-Boland et al., 2001). A variety of DNA-based subtyping methods, such

Abbreviations: CSF, cerebrospinal fluid; MLST, multilocus sequence typing; RAPD, randomly amplified polymorphic DNA; RTE, ready-to-eat.

†Present address: Department of Veterinary Microbiology and Pathology, Washington State University, 402 Bustad Hall, Pullman, WA 99164-7040, USA. as randomly amplified polymorphic DNA (RAPD), ribotyping, PFGE and amplified-fragment length polymorphisms, have been used for genotyping of L. monocytogenes. Based on these methods, L. monocytogenes strains can be divided into two or three distinct lineages (Borucki et al., 2004; Brosch et al., 1994; Call et al., 2003; Keto-Timonen et al., 2003; Wiedmann et al., 1997; Wiedmann, 2002). Whilst a fully automated system for ribotyping (RiboPrinter microbial characterization system; DuPont Qualicon) allows highly reproducible subtyping of $L$. monocytogenes, ribotyping is relatively costly and can be less discriminatory than PFGE (Aarnisalo et al., 2003; Sauders et al., 2003). PFGE and RAPD show high discriminatory abilities, but the inter-laboratory 
reproducibility of these methods, particularly of RAPD, can be limited (Destro et al., 1996; Zhou \& Jiao, 2004). Although PFGE shows a high level of sensitivity for discrimination of L. monocytogenes strains (Aarnisalo et al., 2003; Yde \& Genicot, 2004) and is often considered to be the gold standard for discriminatory ability (Sauders et al., 2003), it may also sometimes detect small genetic differences that may not be epidemiologically significant (Tenover et al., 1995). Furthermore, DNA fragment size-based subtyping methods (such as PFGE and RAPD) are difficult to standardize and, as a consequence, the ease of exchanging and comparing subtype data among laboratories can be severely limited.

DNA sequencing-based methods are increasingly being developed and used for subtyping and characterizing bacterial isolates. The advantages of DNA sequence-based subtyping methods over DNA fragment size-based typing methods include their ability to generate unambiguous data that are portable through web-based databases and that can be used for phylogenetic analyses (Chan et al., 2001; Enright et al., 2001). Cai et al. (2002) showed that actA, a virulence gene that encodes a protein facilitating cell-to-cell spread of $L$. monocytogenes between host cells, is highly polymorphic among L. monocytogenes isolates; sequencing of actA can thus be used to differentiate L. monocytogenes strains effectively. Specifically, sequencing of the $600 \mathrm{bp} 3^{\prime}$-terminal region of act $A$ allowed discrimination of the $15 \mathrm{~L}$. monocytogenes isolates used into 13 sequence types (Cai et al., 2002).

The goal of the study reported here was to define the phylogenetic diversity of L. monocytogenes strains found in China by using DNA sequencing-based subtyping methods and to characterize the virulence potential of selected strains by using a tissue-culture assay with human intestinal epithelial cells. In total, 40 L. monocytogenes isolates obtained from food and sewage samples and from two human listeriosis cases in China were characterized by DNA sequencing of a 597 bp 3' fragment of actA. Whilst multilocus sequence typing (MLST) methods have been reported for $L$. monocytogenes (Cai et al., 2002), sequencing of a single, highly variable gene was chosen as a highly economical alternative to MLST methods. Phylogenetic analyses and comparisons of partial actA sequences for Chinese L. monocytogenes isolates with previously reported actA sequences for L. monocytogenes isolates collected in the USA showed that L. monocytogenes isolates found in China represent the same two major L. monocytogenes lineages found previously among isolates from the USA and Europe (Ericsson et al., 2000; Rasmussen et al., 1995; Wiedmann et al., 1997). Lineage I isolates showed higher invasiveness and cell-to-cell spread characteristics than lineage II isolates, consistent with the predominance of lineage I strains among human cases (Wiedmann et al., 1997; Wiedmann, 2002).

\section{METHODS}

L. monocytogenes strains. In total, 38 L. monocytogenes isolates were obtained from food products $(n=32)$ and sewage samples $(n=6)$ collected in China (Table 1). In addition, two L. monocytogenes isolates from human listeriosis cases that occurred in China were included in this study (Table 1).

The actA sequences for L. monocytogenes isolates collected predominantly in the USA were obtained from Cai et al. (2002). Eleven of the 15 isolates used by Cai et al. (2002) represented human and food isolates from the USA and one isolate was obtained from Europe (FSL X1-002). The geographical origins of the remaining three isolates (FSL J1-022, FSL W1-110 and FSL W1-111) were not available.

PCR. PCR primers were designed based on the published nucleotide sequence for actA (GenBank accession no. NC_003210). Primers 01 (5' GCTGATTTAAGAGATAGAGGAACA-3') and 02 (5'-TTTATGTGGT TATTTGCTGTC-3') amplified an 827 bp DNA fragment that corresponded to the $3^{\prime}$ end of actA. Primers were synthesized by TaKaRa Biotechnology Co. PCR was performed in $100 \mu \mathrm{l}$ reaction volumes containing $96 \mu \mathrm{l}$ PCR mastermix ( $4 \mu \mathrm{l}$ each primer at $12.5 \mu \mathrm{M}, 10 \mu \mathrm{l}$ $10 \times$ PCR buffer, $6.0 \mu \mathrm{l} 25 \mathrm{mM} \mathrm{MgCl}_{2}, 5 \cdot 0 \mu \mathrm{l} 1 \mathrm{mM}$ dNTP mix, $67 \mu \mathrm{l}$ $\left.\mathrm{ddH}_{2} \mathrm{O}\right)$ and $4 \mu \mathrm{L}$. monocytogenes cell lysate. Thermocycling conditions included an initial hold of $2 \mathrm{~min}$ at $94{ }^{\circ} \mathrm{C}$, followed by 35 cycles of $94{ }^{\circ} \mathrm{C}$ for $1 \mathrm{~min}, 50{ }^{\circ} \mathrm{C}$ for $1 \mathrm{~min}$ and $72^{\circ} \mathrm{C}$ for $1 \mathrm{~min}$. A final extension step of $72{ }^{\circ} \mathrm{C}$ for $10 \mathrm{~min}$ was followed by a hold at $4{ }^{\circ} \mathrm{C}$.

Purification and sequencing of PCR products. A $3 \mu$ laliquot of each PCR product was run on a $1.5 \%$ agarose gel to confirm successful amplification of $a c t A$. The remaining $97 \mu \mathrm{l}$ of each PCR product was purified by using a QIAquick PCR Purification kit (Qiagen). DNA sequencing was performed at TaKaRa Biotechnology Co., using both PCR primers to obtain forward and reverse sequences. Where necessary, PCR sequencing was repeated to confirm DNA sequence polymorphisms.

Sequence analysis and construction of a phylogenetic tree. Partial actA sequences obtained for the 40 Chinese L. monocytogenes isolates were aligned with the corresponding actA sequences for the 15 isolates reported by Cai et al. (2002) by using MEGALIGN in the Lasergene software package (DNAStar). Neighbour-joining trees were constructed by using MEGA v. 2.1 (Kumar et al., 2001).

Tissue-culture invasion assay and intracellular growth assays. Enterocyte-like Caco- 2 cells (obtained from P. Cossart, Institut Pasteur, Paris, France) were cultured in Dulbecco's modified Eagle's medium (DMEM) supplemented with $15 \%$ heat-inactivated $\left(30 \mathrm{~min}\right.$ at $56{ }^{\circ} \mathrm{C}$ ) fetal bovine serum (FBS) and $10 \mu \mathrm{g}$ gentamicin $\mathrm{ml}^{-1}$. For invasion and intracellular growth assays, Caco- 2 cells were trypsinized and seeded into six-well plates at a concentration of $1 \times 10^{6}$ cells per well. Cells were grown without gentamicin to form a monolayer $\left(72-76 \mathrm{~h}\right.$ at $\left.37^{\circ} \mathrm{C}\right)$ in DMEM supplemented with $15 \%$ heat-inactivated FBS. The medium was changed every 24 h. L. monocytogenes isolates were grown on brainheart infusion (BHI) broth (Difco) at $37^{\circ} \mathrm{C}$ for $24 \mathrm{~h}$ prior to infection. Bacteria were harvested, adjusted to a concentration of approximately $3 \times 10^{6}$ bacteria $\mathrm{ml}^{-1}$ in DMEM and added to each well, resulting in an m.o.i. of approximately 30 bacteria per host cell. Following $2 \mathrm{~h}$ incubation at $37^{\circ} \mathrm{C}$, cells were washed three times with PBS to remove non-adherent bacteria. To kill extracellular bacteria, $2 \mathrm{ml}$ DMEM containing gentamicin $\left(10 \mu \mathrm{g} \mathrm{ml}^{-1}\right)$ was added to the wells and the cells were then incubated for $1.5 \mathrm{~h}$ at $37^{\circ} \mathrm{C}$ with $5 \% \mathrm{CO}_{2}$. Cells were then lysed with $1 \mathrm{ml}$ ice-cold $0 \cdot 1 \%$ Triton X-100 and the number of viable intracellular bacteria was determined by plating serial dilutions onto BHI agar plates. Invasion efficiency for each isolate was calculated as the ratio of the number of bacteria internalized at $3.5 \mathrm{~h}$ post-infection to the number of bacteria used for infection. To measure intracellular growth, DMEM supplemented with $5 \mu$ g gentamicin $\mathrm{ml}^{-1}$ was used as the growth medium after infection and infected cells were incubated for $19.5 \mathrm{~h}$ after removal of extracellular bacteria (Van Langendonck et al., 1998). The number of intracellular bacteria was then enumerated at $21.5 \mathrm{~h}$ post-infection as described above and intracellular doubling 
Table 1. Chinese L. monocytogenes strains used in this study

\begin{tabular}{|c|c|c|c|}
\hline Strain & Source & Lineage & act $A$ sequence type \\
\hline $\operatorname{Lm} 1579$ & Human CSF & $\mathrm{I}$ & 3 \\
\hline Lm1191 & Human CSF & IIA & 14 \\
\hline Lm03062501 & Fresh fish & I & 1 \\
\hline Lm03041601 & Fresh fish & I & 5 \\
\hline Lm03061101 & Fresh fish & IIA & 13 \\
\hline Lm03040701 & Fresh fish & IIA & 14 \\
\hline Lm03033101 & Fresh fish & IIA & 14 \\
\hline Lm03041602 & Fresh fish & IIA & 14 \\
\hline Lm03042101 & Fresh fish & IIB & 10 \\
\hline Lm03060601 & Fresh fish & IIB & 9 \\
\hline Lm03061802 & Fresh meat & I & 1 \\
\hline Lm03042102 & Fresh meat & I & 1 \\
\hline Lm03042103 & Fresh meat & I & 1 \\
\hline Lm03061803 & Fresh meat & I & 2 \\
\hline Lm03062502 & Fresh meat & I & 3 \\
\hline Lm03040702 & Fresh meat & I & 4 \\
\hline Lm03032002 & Fresh meat & IIB & 8 \\
\hline Lm03041105 & Fresh meat & IIA & 11 \\
\hline Lm03032001 & Fresh meat & IIA & 14 \\
\hline Lm03061801 & Fresh meat & IIA & 14 \\
\hline Lm03032102 & Dairy product & IIA & 14 \\
\hline Lm03060602 & Dairy product & IIB & 8 \\
\hline Lm03041501 & Vegetable & I & 3 \\
\hline Lm03061103 & Vegetable & I & 6 \\
\hline Lm03040704 & RTE food & I & 1 \\
\hline Lm03061104 & RTE food & I & 7 \\
\hline Lm03061808 & RTE food & IIA & 14 \\
\hline Lm03062505 & RTE food & IIA & 12 \\
\hline Lm03041603 & RTE food & IIA & 14 \\
\hline Lm03041102 & RTE food & IIA & 14 \\
\hline Lm03042104 & RTE food & IIB & 10 \\
\hline Lm03032101 & RTE food & IIB & 10 \\
\hline Lm03060604 & RTE food & IIB & 10 \\
\hline Lm03061807 & RTE food & IIB & 10 \\
\hline Lm03060104 & Sewage & I & 6 \\
\hline Lm03060603 & Sewage & IIA & 14 \\
\hline Lm03062503 & Sewage & IIB & 8 \\
\hline Lm03040703 & Sewage & IIB & 10 \\
\hline Lm03040705 & Sewage & IIB & 10 \\
\hline Lm03061102 & Sewage & IIB & 10 \\
\hline
\end{tabular}

times between 3.5 and $21.5 \mathrm{~h}$ post-infection were calculated. In total, 24 selected isolates, representing 12 lineage I and 12 lineage II isolates (see Table 3), were tested in duplicate on two separate occasions.

\section{RESULTS}

\section{Human listeriosis cases}

The two human L. monocytogenes were isolated in April 2001 from the cerebrospinal fluid (CSF) of two patients showing symptoms of meningitis. Both patients showed high temperatures $\left(39.4\right.$ and $38.8{ }^{\circ} \mathrm{C}$, respectively). The first patient was a 49-year-old male; symptoms in this patient included vomiting, drowsiness, seizures, headache and dislike of light. The second patient was a 35-year-old female, who showed symptoms of high fever, headache, stiff neck, nausea, vomiting, discomfort when looking into bright lights, confusion and sleepiness.

\section{Phylogenetic analysis of partial act $A$ sequences}

act $A$ sequences, encompassing a total of $597 \mathrm{bp}$ at the $3^{\prime}$ end of actA, were obtained for all $40 \mathrm{~L}$. monocytogenes isolates from China. A neighbour-joining tree constructed by using these 40 act $A$ sequences, as well as corresponding partial act $A$ sequences obtained by Cai et al. (2002), showed that these 
sequences could be separated into two major lineages (Fig. 1), consistent with the major lineages described previously (Wiedmann et al., 1997). Lineage II could be separated further into two clusters, IIA and IIB (Fig. 1).

\section{Distribution of actA sequence types and L. monocytogenes lineages among US and Chinese isolates}

Among the $40 \mathrm{~L}$. monocytogenes isolates obtained from China, 14 grouped into lineage I and 26 grouped into lineage II. Grouping of L. monocytogenes into clusters IIA and IIB was also found among both Chinese and US L. monocytogenes isolates. No Chinese L. monocytogenes isolates grouped with the lineage III isolates included in the 15 isolates used by Cai et al. (2002). The two human clinical isolates collected in China (Lm1579 and Lm1191) grouped into lineages I and II, respectively. All Chinese isolates from ready-to-eat (RTE) food products, except Lm03040704 and Lm03061104, grouped into lineage II (Table 1). Isolates from fresh food products were found in both lineages, with 10 isolates belonging to lineage I and 12 isolates belonging to lineage II.

In total, 14 act $A$ sequence types could be differentiated among the $40 \mathrm{~L}$. monocytogenes isolates from China. Two lineage I actA sequence types (10 and 14; Table 1) were represented among both Chinese and US isolates.

\section{Nucleotide and deduced amino acid sequence similarities among actA $3^{\prime}$-end sequences}

Nucleotide and deduced ActA amino acid sequences for the 597 bp 3 '-end sequences of actA were compared for the 40 Chinese L. monocytogenes isolates, as well as for the 15 isolates reported by Cai et al. (2002). Nucleotide sequence similarities within clusters IIA and IIB varied from $99 \cdot 7$ to $100 \%$ and from $99 \cdot 3$ to $100 \%$, respectively. Nucleotide sequence similarities within lineage I and lineage II isolates ranged from $98 \cdot 8$ to $100 \%$ and from $90 \cdot 8$ to $100 \%$, respectively. Nucleotide sequence similarity between different lineages varied from $89 \cdot 4$ to $96 \cdot 3 \%$ ( $89 \cdot 4$ to $90 \cdot 3 \%$ between lineages I and II; $90 \cdot 5$ to $96 \cdot 3 \%$ between lineages I and III; $87 \cdot 1$ to $90 \cdot 3 \%$ between lineages II and III).

Amino acid sequence similarities within lineages I, II and III ranged from 97.5 to $100,98.0$ to 100 and 91.5 to $98.5 \%$, respectively. Amino acid sequence similarities between lineages I and II, I and III, and II and III ranged from $84 \cdot 3$ to $85.9 \%$, from 83.8 to $85.9 \%$ and from 84.8 to $85.4 \%$, respectively. Nineteen amino acid sites showed consistent differences among lineages I, II and III (Table 2).

\section{Caco-2 cell invasion and intracellular growth phenotypes for selected lineage I and II isolates}

The ability of 12 lineage I and 12 lineage II isolates to invade and multiply in intestinal epithelial cells was evaluated by using an in vitro model with human enterocyte-like Caco-2 cells (Table 3). The mean invasion efficiency for lineage I isolates $(10 \cdot 2 \%)$ was significantly higher $(t$-test; $P<0 \cdot 05)$

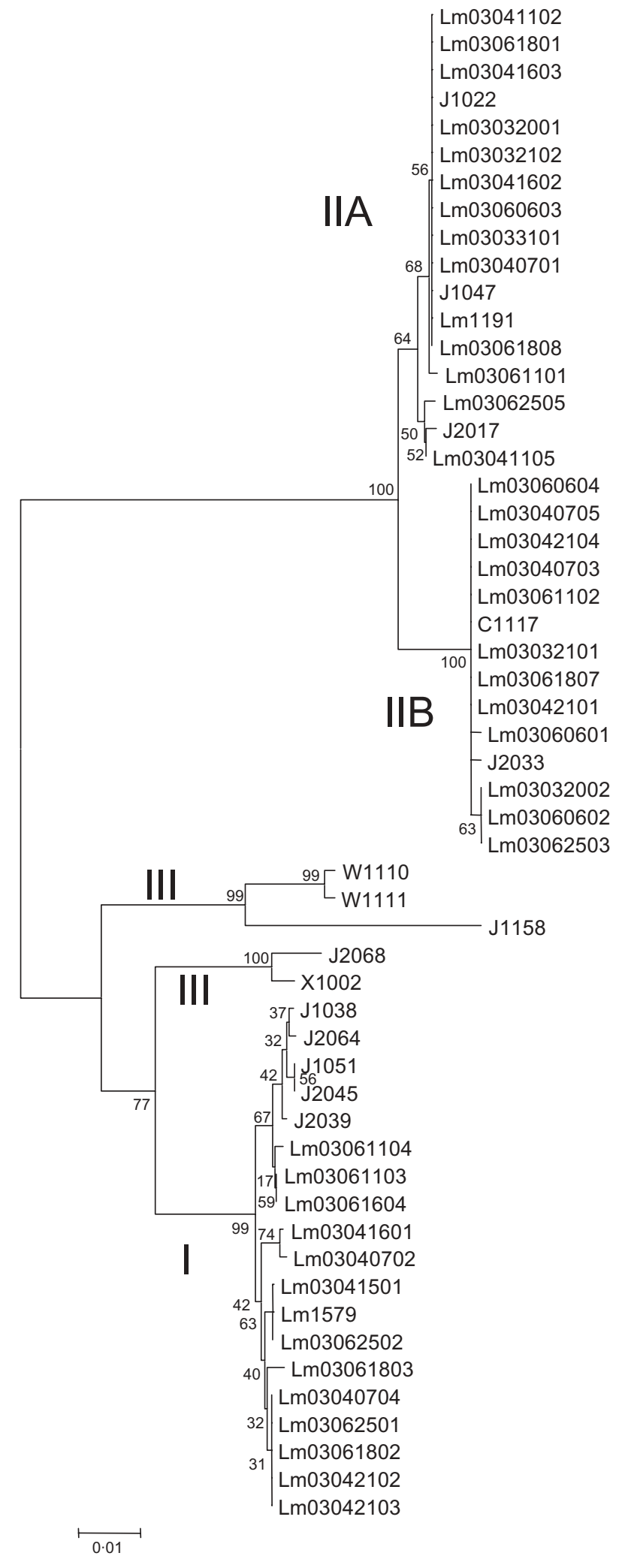

Fig. 1. Unrooted neighbour-joining tree for partial actA sequences from Chinese L. monocytogenes isolates and from Cai et al. (2002). For isolate designations, refer to Table 1 and, for five-digit designations, to Cai et al. (2002), e.g. J1047 represents FSL J1-047 in the study by Cai et al. (2002). 
Table 2. Amino acid residues that differ consistently among L. monocytogenes lineages

\begin{tabular}{|c|c|c|c|c|c|c|c|c|c|c|c|c|c|c|c|c|c|c|c|}
\hline \multirow[t]{2}{*}{ Lineage } & \multicolumn{19}{|c|}{ Amino acid residue } \\
\hline & 435 & 447 & 466 & 470 & 489 & 493 & 504 & 505 & 506 & 508 & 519 & 522 & 523 & 535 & 537 & 541 & 545 & 574 & 594 \\
\hline I & $\mathrm{T}$ & $\mathrm{P}$ & I & $\mathrm{N}$ & G & A & $\mathrm{N}$ & $\mathrm{R}$ & $\mathrm{P}$ & $\mathrm{D}$ & $\mathrm{U}$ & $\mathrm{R}$ & $\mathrm{N}$ & $\mathrm{U}$ & G & $\mathrm{P}$ & $\mathrm{U}$ & $\mathrm{U}$ & $\mathrm{U}$ \\
\hline II & $S$ & S & $\mathrm{P}$ & $\mathrm{K}$ & $\mathrm{R}$ & $\mathrm{T}$ & $\mathrm{K}$ & Q & S & $\mathrm{N}$ & A & $S$ & $\mathrm{D}$ & M & $\mathrm{E}$ & S & $\mathrm{A}$ & G & $\mathrm{F}$ \\
\hline III & S & $\mathrm{P}$ & I & $\mathrm{N}$ & G & A & $\mathrm{N}$ & Q & $\mathrm{P}$ & $\mathrm{D}$ & $\mathrm{U}$ & $\mathrm{R}$ & $\mathrm{N}$ & M & G & $\mathrm{P}$ & $\mathrm{U}$ & $\mathrm{U}$ & $\mathrm{U}$ \\
\hline
\end{tabular}

Table 3. Invasion efficiency and multiplication rate of selected lineage I and II isolates in Caco-2 cells

All data represent the mean of two independent experiments.

\begin{tabular}{|c|c|c|c|c|c|c|}
\hline Strain & Lineage & $\begin{array}{l}\text { No. bacteria } \\
\text { deposited }\end{array}$ & $\begin{array}{l}\text { No. intracellular } \\
\text { bacteria at } 3.5 \mathrm{~h}\end{array}$ & $\begin{array}{c}\text { Invasion efficiency } \\
(\%)\end{array}$ & $\begin{array}{c}\text { No. intracellular } \\
\text { bacteria at } 21 \mathrm{~h}\end{array}$ & $\begin{array}{c}\text { Intracellular doubling } \\
\text { time }(\mathbf{h})\end{array}$ \\
\hline Lm03061104 & I & $2.05 \times 10^{7}$ & $1.86 \times 10^{6}$ & $9 \cdot 1$ & $9 \cdot 8 \times 10^{7}$ & $3 \cdot 06$ \\
\hline Lm03060104 & I & $1.98 \times 10^{7}$ & $1.83 \times 10^{6}$ & $9 \cdot 2$ & $1.15 \times 10^{8}$ & $2 \cdot 93$ \\
\hline Lm03061103 & I & $2 \cdot 01 \times 10^{7}$ & $1.58 \times 10^{6}$ & $7 \cdot 9$ & $1.32 \times 10^{8}$ & $2 \cdot 74$ \\
\hline $\operatorname{Lm} 1579$ & I & $2.25 \times 10^{7}$ & $2 \cdot 17 \times 10^{6}$ & $9 \cdot 6$ & $2.08 \times 10^{8}$ & $2 \cdot 66$ \\
\hline Lm03062502 & I & $2 \cdot 18 \times 10^{7}$ & $2.30 \times 10^{6}$ & $10 \cdot 5$ & $1.96 \times 10^{8}$ & $2 \cdot 73$ \\
\hline Lm03041501 & I & $1.85 \times 10^{7}$ & $2.32 \times 10^{6}$ & $12 \cdot 5$ & $1.82 \times 10^{8}$ & $2 \cdot 78$ \\
\hline Lm03061601 & I & $1.97 \times 10^{7}$ & $2 \cdot 42 \times 10^{6}$ & $12 \cdot 2$ & $1.92 \times 10^{8}$ & $2 \cdot 77$ \\
\hline Lm03040702 & I & $2 \cdot 12 \times 10^{7}$ & $2 \cdot 21 \times 10^{6}$ & $10 \cdot 4$ & $1.80 \times 10^{8}$ & $2 \cdot 76$ \\
\hline Lm03061802 & I & $2 \cdot 40 \times 10^{7}$ & $2.30 \times 10^{6}$ & $9 \cdot 50$ & $9 \cdot 25 \times 10^{7}$ & $3 \cdot 28$ \\
\hline Lm03062501 & I & $2.28 \times 10^{7}$ & $1.44 \times 10^{6}$ & $6 \cdot 60$ & $1.40 \times 10^{8}$ & $2 \cdot 65$ \\
\hline Lm03040704 & I & $2 \cdot 19 \times 10^{7}$ & $2.72 \times 10^{6}$ & $12 \cdot 32$ & $1.66 \times 10^{8}$ & $2 \cdot 95$ \\
\hline Lm03042102 & I & $2.32 \times 10^{7}$ & $2.93 \times 10^{6}$ & $12 \cdot 60$ & $2.36 \times 10^{8}$ & $2 \cdot 76$ \\
\hline Lm1191 & II & $2.08 \times 10^{7}$ & $1.20 \times 10^{6}$ & $5 \cdot 8$ & $5.61 \times 10^{7}$ & $3 \cdot 15$ \\
\hline Lm03061101 & II & $2 \cdot 10 \times 10^{7}$ & $8.31 \times 10^{4}$ & $0 \cdot 39$ & $7.98 \times 10^{5}$ & $5 \cdot 36$ \\
\hline Lm03061808 & II & $1.99 \times 10^{7}$ & $6.81 \times 10^{4}$ & $0 \cdot 34$ & $8.58 \times 10^{5}$ & $4 \cdot 79$ \\
\hline Lm03041602 & II & $2.00 \times 10^{7}$ & $5.32 \times 10^{4}$ & $0 \cdot 27$ & $5.98 \times 10^{5}$ & $5 \cdot 01$ \\
\hline Lm03040701 & II & $1.89 \times 10^{7}$ & $7 \cdot 30 \times 10^{4}$ & $0 \cdot 38$ & $8.60 \times 10^{5}$ & $4 \cdot 92$ \\
\hline Lm03032102 & II & $2.22 \times 10^{7}$ & $7 \cdot 80 \times 10^{4}$ & $0 \cdot 35$ & $7 \cdot 30 \times 10^{5}$ & $5 \cdot 42$ \\
\hline Lm03032001 & II & $2 \cdot 16 \times 10^{7}$ & $7 \cdot 70 \times 10^{4}$ & $0 \cdot 36$ & $2.00 \times 10^{5}$ & $12 \cdot 71$ \\
\hline Lm03041603 & II & $1.99 \times 10^{7}$ & $6.20 \times 10^{4}$ & $0 \cdot 31$ & $4.85 \times 10^{5}$ & $5 \cdot 90$ \\
\hline Lm03041102 & II & $2 \cdot 18 \times 10^{7}$ & $9.00 \times 10^{4}$ & $0 \cdot 41$ & $1 \cdot 19 \times 10^{6}$ & $4 \cdot 70$ \\
\hline Lm03060603 & II & $1.98 \times 10^{7}$ & $4.80 \times 10^{4}$ & $0 \cdot 24$ & $2 \cdot 30 \times 10^{5}$ & $7 \cdot 74$ \\
\hline Lm03033101 & II & $2 \cdot 13 \times 10^{7}$ & $1 \cdot 10 \times 10^{5}$ & $0 \cdot 51$ & $1.20 \times 10^{6}$ & $5 \cdot 08$ \\
\hline Lm03061801 & II & $2 \cdot 28 \times 10^{7}$ & $8.60 \times 10^{4}$ & $0 \cdot 37$ & $1.10 \times 10^{6}$ & $4 \cdot 76$ \\
\hline
\end{tabular}

than that for lineage II isolates $(0 \cdot 8 \%)$. The mean intracellular doubling time for lineage I isolates $(3 \cdot 2 \mathrm{~h})$ was significantly shorter ( $t$-test, $P<0 \cdot 05)$ than that for lineage II isolates $(5 \cdot 8 \mathrm{~h})$.

\section{DISCUSSION}

Human listeriosis is generally caused by consumption of RTE foods that are stored for extended periods of time at refrigeration temperatures and that permit the growth of the causative agent, L. monocytogenes. Food-consumption patterns in China are undergoing rapid changes and more regular consumption of refrigerated-storage RTE foods may increase the risk of human listeriosis in China. In order to understand the epidemiology and ecology of L. monocyto- genes in China in more detail, we characterized $38 \mathrm{~L}$. monocytogenes isolates obtained from food and sewage samples collected in China, as well as two isolates from human listeriosis cases that occurred in China. All isolates were characterized into molecular subtypes by using DNA sequencing of a $597 \mathrm{bp}$ fragment of the L. monocytogenes virulence gene actA. We showed that sequencing of this single, highly variable gene represents an economical alternative to more expensive subtyping methods (e.g. MLST or PFGE). Phylogenetic and subtype analyses showed that the predominant $L$. monocytogenes lineages reported previously among isolates obtained from foods and human clinical cases in Europe and the USA are also present in China. Changing food-consumption and -distribution patterns in China, with increased consumption of RTE foods with extended refri- 
gerated storage times, will thus probably result in an increased number of human listeriosis cases in this country. We also showed that isolates belonging to lineage I expressed, on average, a significantly higher ability to invade and multiply within human intestinal epithelial cells than lineage II isolates, further supporting the suggestion that lineage I isolates may be characterized by enhanced virulence.

\section{Partial actA sequencing represents an economical and discriminatory subtyping and lineage- classification method for $L$. monocytogenes}

Whilst a variety of molecular subtyping methods for $L$. monocytogenes have been described and are used for scientific studies and surveillance purposes, many methods are expensive and time-consuming and, thus, do not lend themselves to routine application for subtyping of large numbers of isolates in many public-health systems. In addition, many of the commonly used DNA banding pattern-based subtyping methods (e.g. ribotyping, PFGE and RAPD) are difficult to standardize and do not provide information suitable for evolutionary analyses. Initial data reported by Cai et al. (2002) showed that DNA sequencing of a $600 \mathrm{bp}$ fragment representing the $3^{\prime}$ end of actA could differentiate $15 \mathrm{~L}$. monocytogenes isolates, representing eight EcoRI ribotypes, into 13 act $A$ sequence types. Thus, actA sequencing, as used here, provides higher subtype discrimination than EcoRI ribotyping, a commonly used molecular subtyping method for L. monocytogenes (Aarnisalo et al., 2003; Mereghetti et al., 2002; Nadon et al., 2001; Wiedmann et al., 1997). Discrimination of $40 \mathrm{~L}$. monocytogenes isolates collected in China into 14 act $A$ sequence types further supports the use of act $A$ sequencing as a sensitive molecular subtyping method.

Our data also showed that DNA sequencing of the $3^{\prime}$ portion of actA not only provides sensitive subtype discrimination, but also allows for evolutionarily meaningful lineage classification of L. monocytogenes isolates. Phylogenetic analyses of partial actA sequences for the 40 Chinese L. monocytogenes isolates and the corresponding actA sequences for the 15 isolates reported by Cai et al. (2002) showed a clear separation of $L$. monocytogenes into the previously reported two main L. monocytogenes lineages, I and II (Fig. 1) (Call et al., 2003; Ericsson et al., 2000; Wiedmann et al., 1997). These lineages correlated with $L$. monocytogenes serotypes: lineage I comprised serotypes $1 / 2 \mathrm{~b}, 3 \mathrm{~b}, 3 \mathrm{c}$ and $4 \mathrm{~b}$ and lineage II comprised serotypes $1 / 2 \mathrm{a}, 1 / 2 \mathrm{c}$ and $3 \mathrm{a}$ (Nadon et al., 2001). In addition, we showed that isolates representing lineage III clearly formed two separate groups that were closer to lineage I than to lineage II isolates in an actA neighbour-joining tree (Fig. 1). Thus, actA sequencing allowed reliable isolate classification into the three L. monocytogenes lineages that have previously been defined by a variety of other subtyping methods, including PFGE (Brosch et al., 1994; Chasseignaux et al., 2001), ribotyping (Wiedmann et al., 1997) and DNA sequence data for $\operatorname{sigB}$ (Moorhead et al., 2003), flaA, iap and hly (Rasmussen et al., 1995).

Three other L. monocytogenes virulence genes ( $h l y, p l c A$ and
inlA) have previously been characterized in two serovar $4 \mathrm{~b}$ strains and two serovar $1 / 2 \mathrm{~b}$ strains (Vines \& Swaminathan, 1998), which belong to lineage I (Nadon et al., 2001). The nucleotide and amino acid similarities among these lineage I strains and strains of serovars $1 / 2 \mathrm{a}$ and $1 / 2 \mathrm{c}$, which belong to lineage II (Nadon et al., 2001), ranged from $97 \cdot 3$ to $98 \cdot 8 \%$ and from $97 \cdot 8$ to $99 \cdot 4 \%$, respectively. In the present study, the corresponding similarity values among lineage I and II isolates were 89.4 to $90.3 \%$ and 84.3 to $85.9 \%$, respectively. This indicates that partial act $A$ sequencing may be better suited to differentiation and classification of L. monocytogenes into lineages than sequencing of other virulence genes ( $h l y, p l c A$ and inlA). Whilst actA sequencing allowed the differentiation of two clusters within lineage II (IIA and IIB), similar subdivisions could not be defined based on inlB sequencing (Ericsson et al., 2000), further supporting the suggestion that partial sequencing of the actA gene may provide a more sensitive subtyping and lineage classification than other virulence genes. A study by Moorhead et al. (2003) also showed that L. monocytogenes could be divided into three lineages based on the nucleotide sequence for $\operatorname{sig} B$, which encodes a stress-responsive alternative sigma factor in L. monocytogenes. The deduced sigma $B$ amino acid sequence, however, was highly conserved and showed only one polymorphic site (Phe216Tyr) (Moorhead et al., 2003). By comparison, we have shown that the deduced protein sequence of the partial actA gene is highly polymorphic, with 19 consistent amino acid differences between lineages I and II. These data not only support the suitability of actA sequencing for sensitive L. monocytogenes subtype discrimination, but also show that ActA is a highly polymorphic virulence protein; it is thus tempting to speculate that these polymorphisms may contribute to the proposed virulence differences between strains of lineages I and II (Wiedmann et al., 1997).

\section{L. monocytogenes subtypes found in China and in Europe and North America represent the same lineages and similar clonal groups}

A PubMed search, conducted on 4 April 2004, revealed very limited information available in English-language journals on the epidemiology of listeriosis in China. In particular, no published reports on human listeriosis cases in China were found. This current study not only represents one of the first reports on the L. monocytogenes molecular subtypes present in the food system in China, but also reports the occurrence of two human listeriosis cases in China. Our data show that the two main L. monocytogenes lineages (I and II) that have previously been described among human, food and animal isolates collected in the USA and Europe (Ericsson et al., 2000; Piffaretti et al., 1989; Rasmussen et al., 1995; Wiedmann et al., 1997) also represent the predominant lineages present in China. Similarly to previous studies in other countries (Norton et al., 2001), lineage II strains, which include serotypes $1 / 2 \mathrm{a}$ and $1 / 2 \mathrm{c}$, were found more frequently among RTE foods than lineage I strains. Lineage I strains were predominant among isolates from meats and fresh 
vegetables. None of the L. monocytogenes isolates from China grouped into lineage III, which is not surprising, as Wiedmann et al. (1997) showed that strains of lineage III are rare among isolates from humans and food samples and are predominantly associated with animals; no animal isolates from China were included in this study. Interestingly, two act $A$ sequence types (10 and 14; Table 1) were found among both Chinese isolates and human isolates obtained in the USA (Cai et al., 2002). In summary, our data suggest strongly that the L. monocytogenes strains present in China are very similar to those found in the USA and other countries. L. monocytogenes lineages and clonal groups thus appear to be distributed globally, even in countries that have previously not reported human and animal listeriosis cases.

\section{Virulence potential of isolates representing the genotypically distinct lineages I and II}

The discrepancy between the widespread distribution of $L$. monocytogenes in RTE food products (Inoue et al., 2000; Wiedmann, 2002) and the low incidence of listeriosis (e.g. 2500 cases per year in the USA) (Mead et al., 1999) led to the hypothesis that some L. monocytogenes strains in food products may have attenuated virulence and may thus have limited potential to cause human disease (Hof \& Rocourt, 1992; Rasmussen et al., 1995). Comparative genomics showed that virulent $L$. monocytogenes strains may possess genes that are not present in avirulent isolates, and these markers can be used for PCR assessment of L. monocytogenes virulence (Liu et al., 2003). As the virulence of L. monocytogenes depends critically on the organism's ability to invade human intestinal epithelial cells, we evaluated the ability of selected Chinese lineage I and II L. monocytogenes isolates to invade and multiply within the human enterocyte cell line Caco-2. Our results showed that lineage I isolates had a significantly higher invasion efficiency and significantly faster doubling times than lineage II strains in this cellculture assay. Interestingly, the one human clinical lineage II isolate showed a higher invasion efficiency $(5.8 \%)$ and shorter intracellular doubling time $(3 \cdot 15 \mathrm{~h})$ than the other lineage II isolates (Table 3), which were obtained from food. These findings are consistent with previous studies showing that certain genetic types of L. monocytogenes found commonly in food are less invasive than others to Caco-2 cells (Larsen et al., 2002) and mouse L cells (Norton et al., 2001). As isolates from RTE food products belonged predominantly to lineage II (Table 1), we hypothesize that RTE food products in China may predominantly harbour less invasive L. monocytogenes strains. Possible reasons why RTE food products may harbour more low-virulence strains than fresh food products need to be further investigated.

\section{Conclusions}

Based on and confirming a previous small study on $15 \mathrm{~L}$. monocytogenes isolates (Cai et al., 2002), we have shown that $3^{\prime}$-end partial nucleotide sequencing of actA provides an economical and sensitive approach for subtype and lineage classification of $L$. monocytogenes. By using this subtyping approach, we have also shown that $L$. monocytogenes isolates from foods and environmental samples collected in China represent the same major L. monocytogenes genetic lineages that have previously been identified in North America and Europe, including human listeriosis cases. The frequency of these lineages among food isolates was also comparable with that reported in many other countries, with a predominance of lineage II strains among food isolates (Norton et al., 2001). In combination with the first description of two human listeriosis cases in China, our data suggest that changes in food-distribution and -consumption patterns in China and other countries will lead to the emergence of human listeriosis as a food-safety issue, as virulent strains of this pathogen appear to be present in food systems worldwide. Furthermore, our results showed that L. monocytogenes occurring in RTE food products are predominantly lineage II strains and that lineage II strains, on average, may be less virulent than lineage I strains. This phenomenon may partly contribute to the low incidence of human listeriosis, despite the frequent human exposure to L. monocytogenes through contaminated food products.

\section{ACKNOWLEDGEMENTS}

This study was supported by grants from NSFC, the Ministry of Education of PR China and Jiangsu Provincial Government (Contract no. TRAPOYT175, 30425031, BE2003307 and BZ2003031). This work was also supported in part by the National Institutes of Health Award no. R01GM63259 (to M. W.).

\section{REFERENCES}

Aarnisalo, K., Autio, T., Sjoberg, A. M., Lunden, J., Korkeala, H. \& Suihko, M. L. (2003). Typing of Listeria monocytogenes isolates originating from the food processing industry with automated ribotyping and pulsed-field gel electrophoresis. J Food Prot 66, 249-255.

Altimira, J., Prats, N., López, S., Domingo, M., Briones, V., Domínguez, L. \& Marco, A. (1999). Repeated oral dosing with Listeria monocytogenes in mice as a model of central nervous system listeriosis in man. J Comp Pathol 121, 117-125.

Borucki, M. K., Kim, S. H., Call, D. R., Smole, S. C. \& Pagotto, F. (2004). Selective discrimination of Listeria monocytogenes epidemic strains by a mixed-genome DNA microarray compared to discrimination by pulsed-field gel electrophoresis, ribotyping, and multilocus sequence typing. J Clin Microbiol 42, 5270-5276.

Brosch, R., Chen, J. \& Luchansky, J. B. (1994). Pulsed-field fingerprinting of listeriae: identification of genomic divisions for Listeria monocytogenes and their correlation with serovar. Appl Environ Microbiol 60, 2584-2592.

Cai, S., Kabuki, D. Y., Kuaye, A. Y., Cargioli, T. G., Chung, M. S., Nielsen, R. \& Wiedmann, M. (2002). Rational design of DNA sequence-based strategies for subtyping Listeria monocytogenes. J Clin Microbiol 40, 3319-3325.

Call, D. R., Borucki, M. K. \& Besser, T. E. (2003). Mixed-genome microarrays reveal multiple serotype and lineage-specific differences among strains of Listeria monocytogenes. J Clin Microbiol 41, 632-639.

Chan, M.-S., Maiden, M. C. J. \& Spratt, B. G. (2001). Database-driven multi locus sequence typing (MLST) of bacterial pathogens. Bioinformatics 17, 1077-1083.

Chasseignaux, E., Toquin, M.-T., Ragimbeau, C., Salvat, G., Colin, P. \& 
Ermel, G. (2001). Molecular epidemiology of Listeria monocytogenes isolates collected from the environment, raw meat and raw products in two poultry- and pork-processing plants. J Appl Microbiol 91, 888-899.

Destro, M. T., Leitão, M. F. F. \& Farber, J. M. (1996). Use of molecular typing methods to trace the dissemination of Listeria monocytogenes in a shrimp processing plant. Appl Environ Microbiol 62, 705-711.

Enright, M. C., Spratt, B. G., Kalia, A., Cross, J. H. \& Bessen, D. E. (2001). Multilocus sequence typing of Streptococcus pyogenes and the relationships between emm type and clone. Infect Immun 69, 2416-2427.

Ericsson, H., Unnerstad, H., Mattsson, J. G., Danielsson-Tham, M.-L. \& Tham, W. (2000). Molecular grouping of Listeria monocytogenes based on the sequence of the inlB gene. J Med Microbiol 49, 73-80.

Hof, H. \& Rocourt, J. (1992). Is any strain of Listeria monocytogenes detected in food a health risk? Int J Food Microbiol 16, 173-182.

Inoue, S., Nakama, A., Arai, Y. \& 7 other authors (2000). Prevalence and contamination levels of Listeria monocytogenes in retail foods in Japan. Int J Food Microbiol 59, 73-77.

Keto-Timonen, R. O., Autio, T. J. \& Korkeala, H. J. (2003). An improved amplified fragment length polymorphism (AFLP) protocol for discrimination of Listeria isolates. Syst Appl Microbiol 26, 236-244.

Kumar, S., Tamura, K., Jakobsen, I. B. \& Nei, M. (2001). MEGA2: molecular evolutionary genetic analysis software. Bioinformatics $\mathbf{1 7}$, 1244-1245.

Larsen, C. N., Nørrung, B., Sommer, H. M. \& Jakobsen, M. (2002). In vitro and in vivo invasiveness of different pulsed-field gel electrophoresis types of Listeria monocytogenes. Appl Environ Microbiol 68, 56985703.

Liu, D., Ainsworth, A. J., Austin, F. W. \& Lawrence, M. L. (2003). Characterization of virulent and avirulent Listeria monocytogenes strains by PCR amplification of putative transcriptional regulator and internalin genes. J Med Microbiol 52, 1065-1070.

Mead, P. S., Slutsker, L., Dietz, V., McCaig, L. F., Bresee, J. S., Shapiro, C., Griffin, P. M. \& Tauxe, R. V. (1999). Food-related illness and death in the United States. Emerg Infect Dis 5, 607-625.

Mereghetti, L., Lanotte, P., Savoye-Marczuk, V., Marquet-Van Der Mee, N., Audurier, A. \& Quentin, R. (2002). Combined ribotyping and random multiprimer DNA analysis to probe the population structure of Listeria monocytogenes. Appl Environ Microbiol 68, 2849-2857.

Moorhead, S. M., Dykes, G. A. \& Cursons, R. T. (2003). An SNP-based PCR assay to differentiate between Listeria monocytogenes lineages derived from phylogenetic analysis of the sigB gene. J Microbiol Methods $\mathbf{5 5}, 425-432$

Nadon, C. A., Woodward, D. L., Young, C., Rodgers, F. G. \& Wiedmann, M. (2001). Correlations between molecular subtyping and serotyping of Listeria monocytogenes. J Clin Microbiol 39, 2704-2707.
Norton, D. M., Scarlett, J. M., Horton, K., Sue, D., Thimothe, J., Boor, K. J. \& Wiedmann, M. (2001). Characterization and pathogenic potential of Listeria monocytogenes isolates from the smoked fish industry. Appl Environ Microbiol 67, 646-653.

Piffaretti, J.-C., Kressebuch, H., Aeschbacher, M., Bille, J., Bannerman, E., Musser, J. M., Selander, R. K. \& Rocourt, J. (1989). Genetic characterization of clones of the bacterium Listeria monocytogenes causing epidemic disease. Proc Natl Acad Sci U S A 86, 3818-3822.

Rasmussen, O. F., Skouboe, P., Dons, L., Rossen, L. \& Olsen, J. E. (1995). Listeria monocytogenes exists in at least three evolutionary lines: evidence from flagellin, invasive associated protein and listeriolysin $\mathrm{O}$ genes. Microbiology 141, 2053-2061.

Sauders, B. D., Fortes, E. D., Morse, D. L., Dumas, N., Kiehlbauch, J. A., Schukken, Y., Hibbs, J. R. \& Wiedmann, M. (2003). Molecular subtyping to detect human listeriosis clusters. Emerg Infect Dis 9, 672-680.

Tenover, F. C., Arbeit, R. D., Goering, R. V., Mickelsen, P. A., Murray, B. E., Persing, D. H. \& Swaminathan, B. (1995). Interpreting chromosomal DNA restriction patterns produced by pulsed-field gel electrophoresis: criteria for bacterial strain typing. J Clin Microbiol 33, 22332239.

Van Langendonck, N., Bottreau, E., Bailly, S., Tabouret, M., Marly, J., Pardon, P. \& Velge, P. (1998). Tissue culture assays using Caco-2 cell line differentiate virulent from non-virulent Listeria monocytogenes strains. J Appl Microbiol 85, 337-346.

Vázquez-Boland, J. A., Kuhn, M., Berche, P., Chakraborty, T., Domínguez-Bernal, G., Goebel, W., González-Zorn, B., Wehland, J. \& Kreft, J. (2001). Listeria pathogenesis and molecular virulence determinants. Clin Microbiol Rev 14, 584-640.

Vines, A. \& Swaminathan, B. (1998). Identification and characterization of nucleotide sequence differences in three virulence-associated genes of Listeria monocytogenes strains representing clinically important serotypes. Curr Microbiol 36, 309-318.

Wiedmann, M. (2002). Molecular subtyping methods for Listeria monocytogenes. J AOAC Int 85, 524-531.

Wiedmann, M., Bruce, J. L., Keating, C., Johnson, A. E., McDonough, P. L. \& Batt, C. A. (1997). Ribotypes and virulence gene polymorphisms suggest three distinct Listeria monocytogenes lineages with differences in pathogenic potential. Infect Immun 65, 2707-2716.

Yde, M. \& Genicot, A. (2004). Use of PFGE to characterize clonal relationships among Belgian clinical isolates of Listeria monocytogenes. J Med Microbiol 53, 399-402.

Zhou, X. \& Jiao, X. (2004). Investigation of Listeria monocytogenes contamination pattern in local Chinese food market and the tracing of two clinical isolates by RAPD analysis. Food Microbiol 21, 695-702. 